\title{
Numerical investigation of laminar mixing in a coaxial microreactor
}

\author{
By L. DJENIDI AND B. MOGHTADERI \\ School of Engineering, The University of Newcastle, Newcastle, 2308 NSW, Australia
}

(Received 1 November 2005 and in revised form 30 April 2006)

This paper reports on a lattice Boltzmann simulation of laminar mixing in a coaxial microreactor at low Reynolds numbers. The main focus of the study was to compare the effectiveness of a multi-holed baffle plate, a set of $2 \times 2$ square bars, and a flat-plate arrangement on the enhancement of reagent mixing inside the reactor. It was found that all three mixers increased mixing through the mechanism of generating coherent structures, which in turn increased interface contact between the fluid streams. However, for this particular microreactor, the efficiency of each mixer depended on its ability to generate coherent structures with high helicity and thereby inducing a swirl motion to the flow.

\section{Introduction}

The last decade has seen a surge of interest in microfluidic-based devices because of their novel biological engineering applications (Ho \& Tai 1998; Karniadakis \& Beskok 2002; Verpoorte \& Rooij 2003; Stone, Strook \& Ajdari 2004). Of particular interest, are the miniaturized reactors or microreactors (see Ehrfeld, Hessel \& Loww 2000 for a summary on microreactors). These reactors are highly suitable for integration into a wide variety of systems ranging from hydrogen powered fuel cells for mobile and/or onboard applications, such as electrical automobiles, cell phones and notebook computers, to microfluidic devices for medical diagnostics, genetic sequencing, chemical analysis, drug discovery and proteonics (Ehrfeld et al. 2000). Because of their small physical dimensions, microreactors often operate in the laminarflow regime (low Reynolds number) and, thus, mixing of reactive agents within these reactors is mainly governed by molecular diffusion which unfortunately limits the speed of mixing (Nguyen \& Werely 2002).

Enhancement of laminar mixing in microreactors is possible by using either active or passive mixers (Ottino \& Wiggins 2004). Active mixers typically involve moving parts and require energy input. For example, Evensen, Meldrum \& Cunningham (1998) used piezo-ceramic actuators to move air back and forth, thereby inducing mixing. Similarly, Lee et al. (2001) and Deval, Tabeling \& Ho (2002) generated folding and stretching along a channel using an external perturbation to enhance mixing. Suzuki \& Ho (2002) used a magnetic field to induce chaotic mixing in the flow containing macromolecules. The use of ultrasound to enhance mixing has also been reported (Ehrfeld et al. 2000; Nguyen \& Werely 2002).

Passive mixers, on the other hand, do not require input of energy and have no moving parts. Passive mixers are of particular interest in microfluidics because the enhanced mixing can be achieved by proper design of the reactor geometry without the need for additional units. According to molecular diffusion theory, fast mixing within passive mixers can be accomplished by decreasing the mixing path and/or increasing 
(a)
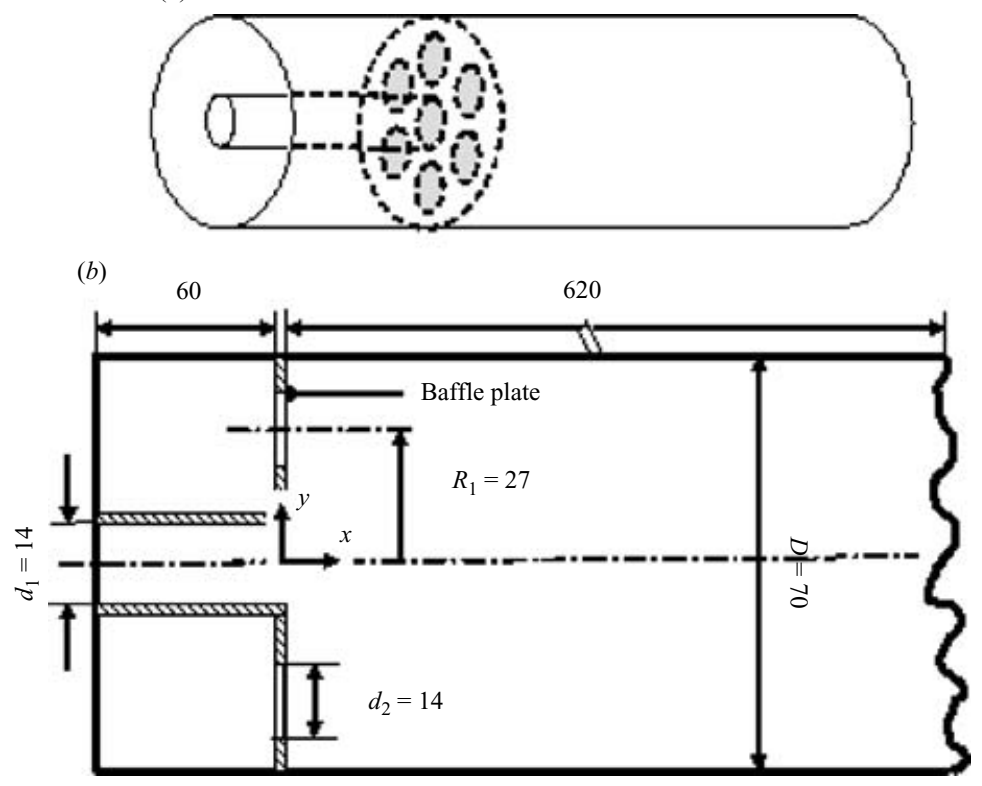

FIGURE 1. Sketch (not to scale) of the coaxial microreactor with the multi-holed baffle plate. Dimensions are in lattice units. $(a)$ Perspective view, $(b)$ side view.

the mixing contact area between the fluids. This is achieved through a series of strategies commonly referred to as streaming mixing (SM). Some of these strategies include contacting, e.g. contact of two sub-streams in a T- or Y-shaped configured channel, collision, injections and atomization, velocity increase and manifold splitting and recombination (see Ehrfeld et al. 2000 for an extensive review on the subject).

It is clear that the choice of the laminar mixing enhancement method can significantly affect the complexity of the reactor design, influencing its geometry and topological properties, and, in turn, its flow structure. One simple laminar mixing method in a microreactor consists of a type of a coaxial jet; a tubular vessel fitted with a fuel inlet tube, located coaxially in the main vessel (see figure 1). While the work on mixing in a coaxial microreactor is not comprehensive, the advantage of the design is of practical interest: mixer and reactor can be the same microfluidic vessel. The mixing occurs at the interface of the two fluids through molecular diffusion. However, while mixing occurs, it is relatively inefficient owing to lack of turbulent mixing. In order to improve mixing, one must increase the interfacial area between the two fluids. Woodfield, Kazuyoshi \& Suzuki (2003) and Moghtaderi, Shames \& Djenidi (2006) showed that fitting a multi-holed baffle plate (figure 1, see also figures 3 and 4), dramatically improves the laminar mixing. The mechanism by which mixing is enhanced is that of stucture generation. The jetflows issuing from the holes create recirculation zones (vortical structures) around the coaxial tube, which promote interaction between the oxidizer and the fuel, which in turn increases the interfacial area, thus enhancing the mixing process. The parametrical study of Woodfield et al. (2003) and the experimental investigation of Moghtaderi et al. (2006) showed that the mixing performance is strongly dependent on the vortical stuctures generated by the baffle plate, which in turn are dependent on several geometrical parameters, i.e. the fuel inlet and main vessel diameters, the location of the baffle plate holes relative 
to the fuel inlet and their diameter. It is likely that other configurations can also be effective streaming mixing.

This present paper reports on an exploratory study of laminar-mixing enhancement in a coaxial microreactor through the use of passive means. Our primary focus is to explore some strategies to generate vortical structures, which can help improve mixing at low Reynolds numbers. The motivation for this work is the need for simple and relatively maintenance-free laminar-mixing passive devices for tubular microreactors. Maintenance can be problematic. For example, one possible problem with the multiholed baffle-plate tubular microreactor is clogging because of the small diameter of the baffle-plate holes. Another issue is blockage due to the baffle plate; this requires extra energy pumping to achieve the same level of oxidizer flow rate as that without the baffle plate. It is then desirable to investigate means for generating vortical structures which can minimize this additional energy input. Also, bearing in mind that a typical diameter size of a microchannel is about $100 \mu \mathrm{m}$, there is a need to keep the reactor geometry as simple as possible for fabrication related reasons. Thus, the present investigation concentrates solely on simple passive devices to enhance mixing.

Numerical simulations were performed using the lattice Boltzmann method (LBM, Benzi, Succi \& Vegassola 1992; Chen \& Doolen 1998; Succi 2001). The tubular microreactor shown in figure 1 was considered as the base geometry. Since the study focuses on mixing though mechanical process (stretching and folding) the same non-reacting, isothermal and incompressible fluid was used for representing both oxidizer and fuel streams. It should be pointed out that although diffusivity ultimately dictates the molecular diffusion rate, it is not an issue in the present study since the process investigated (stretching and folding) is independent of diffusivity.

\section{Numerical procedure}

\subsection{The lattice Boltzmann method}

The numerical simulations were performed using the lattice Boltzmann method (LBM), an alternative numerical method to the classical direct numerical simulations. The LBM is based on kinetic theory. Rather than solving the governing fluid equations (Navier-Stokes equations), the LBM solves the Boltzmann equation on a lattice. The basic idea of the LBM is to construct a simplified kinetic model that incorporates the essential physics of microscopic average properties, which obey the desired (macroscopic) Navier-Stokes equations (Frisch, Hasslacher \& Pomeau 1986). With sufficient symmetry of the lattice, the LBM inherently solves these latter equations with second-order accuracy. For the present calculations, each computational node consisted of a three-dimensional lattice composed of 18 moving particles and a rest particle (lattice model D3Q19, figure 2). The spacings in the three directions between each node are noted as $\Delta x, \Delta y$ and $\Delta z$.

The Boltzmann equation is discretized on that lattice and results in the lattice Boltzmann equation, which governs the time and space variations of the single-particle distribution $f_{i}(\boldsymbol{x}, t)$ at the lattice site $\boldsymbol{x}$ :

$$
f_{i}\left(\boldsymbol{x}+\boldsymbol{e}_{i} \Delta t, t+\Delta t\right)-f_{i}(\boldsymbol{x}, t)=-\frac{\Delta t}{\tau}\left(f_{i}(\boldsymbol{x}, t)-f_{i}^{e q}(\boldsymbol{x}, t)\right) \quad(i=0,1, \ldots, 18),
$$

where $\tau$ is the relaxation time, $\Delta t$ the time step, $\boldsymbol{e}_{i}(=\Delta x / \Delta t)$ is the particle velocity in the $i$-direction and $f_{i}^{e q}$ is the equilibrium single-particle distribution:

$$
f_{i}^{e q}=\rho \omega_{i}\left(1+3\left(\boldsymbol{e}_{i} \cdot \boldsymbol{u}\right)+\frac{9}{2}\left(\boldsymbol{e}_{i} \cdot \boldsymbol{u}\right)^{2}-\frac{3}{2} u^{2}\right),
$$




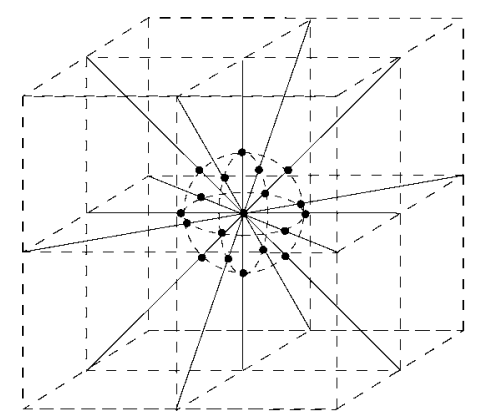

FIGURE 2. D3Q19 square lattice model.

where $\rho\left(=\sum_{i} f_{i}\right)$ is the fluid density, $\boldsymbol{u}\left(\rho \boldsymbol{u}=\sum_{i} f_{i} \boldsymbol{e}_{i}\right)$ is the local fluid velocity and $\omega_{i}$ are the corresponding weights $\left(\omega_{i}=1 / 3\right.$ for $i=0 ; 1 / 18$ for $i=1$ to 6 ; and $1 / 36$ for $i=7$ to $18 ; i=0$ corresponds to the rest particle in the centre of the cubic lattice, $i=1, \ldots, 6$ correspond to the particles on the axis aligned with $x, y$ and $z$, and $i=7, \ldots, 18$ are related to the particles on the diagonal directions). The relaxation time, $\tau$, is related to the kinematic viscosity of the fluid via the relation:

$$
\nu=\frac{2 \tau-1}{6} .
$$

In the above equations $\Delta t=\Delta x=\Delta y=\Delta z=1$. With this setting, $\left|\boldsymbol{e}_{i}\right|=1$, for $i=1$ to 6 , and $\sqrt{2}$ for $i=7$ to 18 ; note that $\left|\boldsymbol{e}_{0}\right|=0$, and the dimensional variables such as, $u, v$ and $\rho$ are expressed in lattice units. The relation between the lattice units and the real dimensions can be obtained by writing $L_{\text {lattice }}=N \times \Delta x_{\text {lattice }}$ and $L_{\text {real }}=N \times \Delta x_{\text {real }}$, which yields $\Delta x_{\text {real }}=\left(L_{\text {real }} / L_{\text {lattice }}\right) \Delta x_{\text {lattice }}$ where $L_{\text {lattice }}$ and $\Delta x_{\text {lattice }}$ are a length scale and the space step in lattice units and $L_{\text {real }}$ and $\Delta x_{\text {real }}$ are a length scale and the space step in real dimensions; $N$ is the number of mesh points in the $x$-direction. In the present case, where the microreactor outer diameter is about $D=300 \mu \mathrm{m}$, $L_{\text {real }} / L_{\text {lattice }}=4.3 \mu \mathrm{m} /$ lattice unit.

The left-hand side of (2.1) is the so-called streaming operation, which means that the particles move to the nearest neighbours along their velocity directions (i.e. they radiate from the centre of the lattice along their velocity direction). The right-hand side is the collision term, here modelled by the BGK collision operator, which describes the redistribution of the particles at each node (for more detail see, for example, Chen \& Doolen 1998; Higuera \& Jimenez 1989; Higuera, Succi \& Benzi 1989). Thus, (2.1) is solved according to these two rules: collision and streaming. The collision step is described by:

$$
f_{i}^{n e w}(\boldsymbol{x}, t)=f_{i}(\boldsymbol{x}, t)-\frac{\Delta t}{\tau}\left(f_{i}(\boldsymbol{x}, t)-f_{i}^{e q}(\boldsymbol{x}, t)\right),
$$

where the $f_{i}^{e q}$ are calculated using (2.2). The streaming is described by

$$
f_{i}\left(\boldsymbol{x}+\boldsymbol{e}_{i} \Delta t, t+\Delta t\right)=f_{i}^{n e w}(\boldsymbol{x}, t) .
$$

The collisions are entirely local, making the LBM efficiently parallelized. At time $t$, the particle distributions are updated based on (2.4); then, at time $t+\Delta t$, the particles propagate according to (2.5).

The choice of the LBM over the classical resolution of the Navier-Stokes equations for the present simulations was motivated by its two important and practical advantages: (i) extreme ease of implementation of complex solid surfaces, and (ii) the 


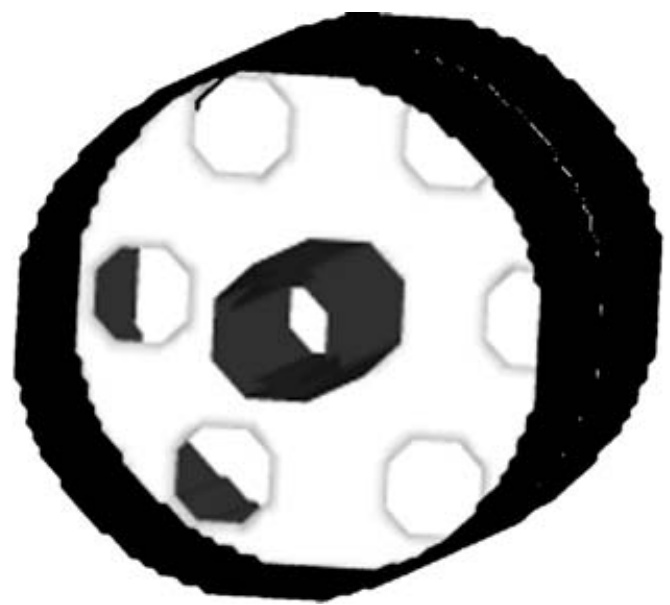

Figure 3. Perspective view of a portion (between the inlet and some distance after the baffle plate) of the microreactor. The multi-holed baffle plate is white, while the inner (fuel) and outer (oxydizer) tubes are black.

local nature of the collision renders the parallelization of any LBM code quite natural and simple. Point (ii) is made possible because, conversely to the resolution of the Navier-Stokes equations, there is no need to solve the Poisson equation for the pressure. This also provides a significant avantage, particularly for fluid-flow complex geometies (Rossi et al. 2005). The code has been successfully used by our group for simulating microchannels (Agrawal, Djenidi \& Antonia 2005) and macro flows (Djenidi 2006).

\subsection{The computational details}

The computational domain for the present work is shown in figure 1 . The microreactor was composed of an outer straight tube and a inner tube with a multi-holed baffle plate fixed as shown. Figure 3 shows a portion of the reactor with a multi-holed baffle plate fitted at the exit plane of the central tube.

The computational uniform Cartesian mesh consisted of $620 \times 74 \times 74$ mesh points with $\Delta x=\Delta y=\Delta z=1 \quad(x$ is the longitudinal direction and $y$ and $z$ the lateral directions, the origin $(0,0,0)$ is on the centreline and at the outlet of the inner tube). Figure 4 shows the computational grid; also illustrated are the baffle plate located at the outlet of the inner tube, the inner tube and the outer wall of the main vessel.

At the inlet of the inner tube, a uniform velocity $\left(U_{\text {in }}=0.05\right.$, and $\left.V_{i n}=W_{i n}=0\right)$ was imposed. At the inlet of the outer cylinder, another uniform velocity is applied $\left(U_{\text {out }}=0.02\right.$, and $\left.V_{\text {out }}=W_{\text {out }}=0\right)$. At the outlet section, the velocty gradient in the streamwise direction is set to zero; note that a convective boundary condition was applied at the outlet and no significant difference between the two outlet conditions was observed in the results. A bounce-back boundary was used to simulate the no-slip condition at the grid elements; when a particle reaches the wall, it bounces back along the same direction. The value of the Knudsen number $\left(=l_{m} / H=v / c_{s} D\right.$; $l_{m}$ and $D$ are the molecular mean free path and the characteristic hydrodynamic length scale, here the outer cylinder diameter; $c_{s}$ is the speed of sound equal to $1 / \sqrt{3}$ ) in the present simulation is about 0.0007 , well below the upper limit $(0.01)$ for which the flow reaches the so-called slip-flow regime. Thus, the bounce-back scheme is well justified here. 


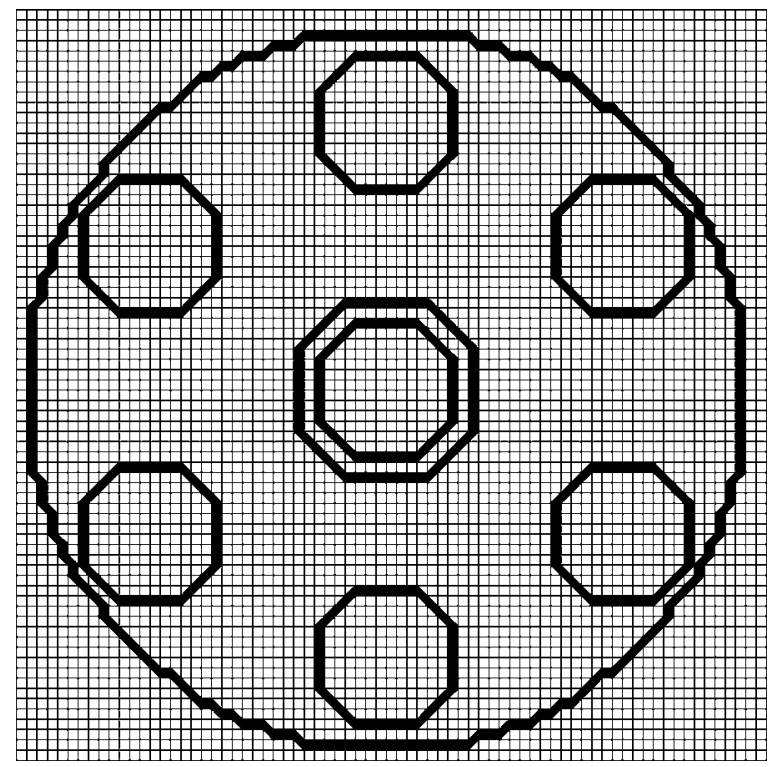

Figure 4. Computational grid in the $(y, z)$-plane; the baffle plate and the inner and outer tubes are also shown. The baffle plate is located at the inner tube outlet (see figure 1).

The Reynolds number, $R_{D}\left(=U_{\text {avg }} D / \nu, U_{\text {avg }}\right.$ is the average velocity in the microreactor), was about 100. This is the same value as that Moghtaderi et al. (2006) had in their microreactor where the flow was laminar. The mass flow rate of the central tube is about $4 \%$ of that of the outer tube. This matches approximately the mass flow rate ratio of the experiment of Moghtaderi et al. (2006). The steady-state solution was reached after about 3000 iterations. However, to remove any remaining effects of the transient regime, the velocity field was saved at the 6000th iteration. Subsequent velocity fields showed no changes.

The calculations were carried out on a dual processor $(2 \times 2.4 \mathrm{GHz}) \mathrm{HP}$ workstation. The collision and streaming steps have been parallelized using the OpenMP procedure. During these steps, each processor worked on half the computational domain.

\section{Results}

\subsection{Microreactor without vortical structure generator}

Figure 5 shows the velocity distributions at two streamwise locations $\left(x / d_{1}=-1\right.$ and 14 ; the centreline of the microreactor is at $y=37$ ). The parabolic shape of the profile distributions indicates that the flow is fully developed in both the inner and outer tubes of the reactor at $x / d_{1}=-1$, as well as at the downstream section in the main vessel $\left(x / d_{1}=14\right)$.

The flow in this latter section recovers quickly to a fully developed state after the merging of the two streams (figure 5): the flow in the main vessel assumes a laminar fully developed profile at $x / d_{1}=14$ which is merely about twice the main vessel diameter. Figure 6 shows the computed velocity field in the plane $(x, y)$ at $z=0$, which compares well with that (not shown here) obtained from micro-PIV measurements of Moghtaderi et al. (2006) and the simulation of Woodfield et al. (2003). Despite the inevitable deviation around the inner tube outlet, the two streams 


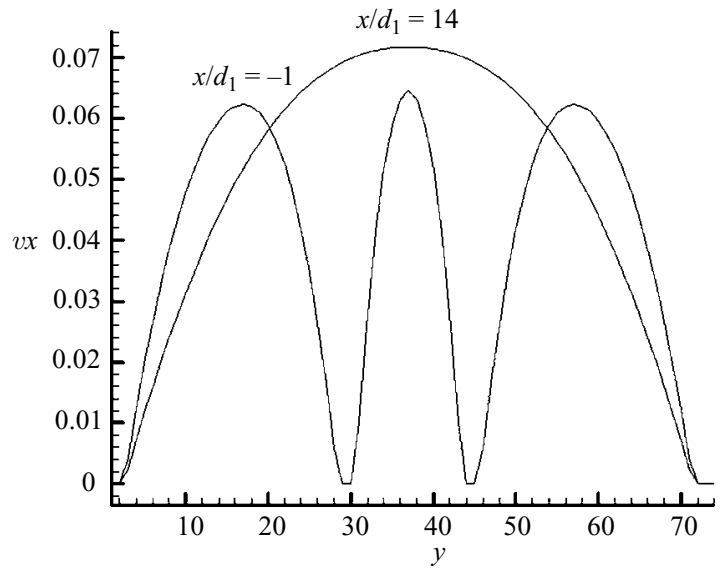

FIGURE 5. Velocity profile at two positions: $x / d_{1}=-1$ (upstream of the inner tube exit) and 14 (downstream of the inner tube exit). The plots are at $z=0$.

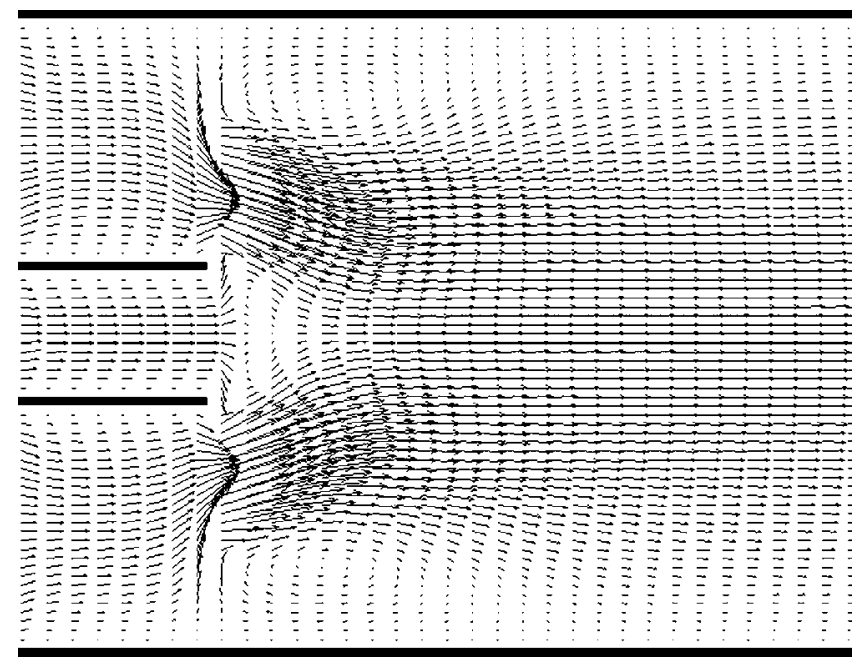

FIGURE 6. Velocity field in the $(x, y)$-plane at $z=0$ without the multi-holed baffle plate.

of fluid do not mix or, at least, very little in the main vessel. Clearly, to improve mixing we must generate transversal flow (flow perpendicular to the main flow direction) which will increase the interfacial area between the two fluids. This is investigated next with the use of passive devices.

\subsection{Microreactor with vortical structure generator}

\subsubsection{Multi-holed baffle plate}

To further assess the validity of the present LBM simulation, a test case with the multi-holed baffle plate mounted in the microreactor was carried out. The multi-holed baffle plate was used by Woodfield et al. (2003) and Moghtaderi et al. (2006) and will provide a reference for the present study. Figure 7 shows an example of the measured (Moghtaderi et al. 2006)) and calculated velocity fields; only a section around the multi-holed baffle plate and for $0<y<37$ is represented. Note the relatively good agreement between the two sets of data, indicating that the present simulations reliably 


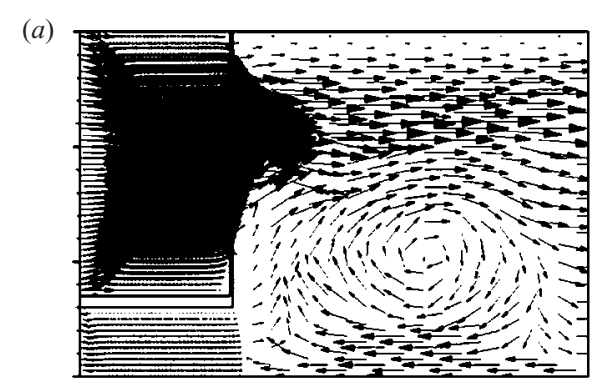

(b)

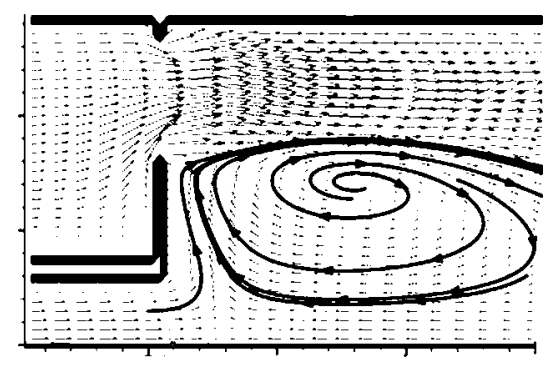

FIGURE 7. Comparison between (a) measurements (Moghtaderi et al. 2006) and (b) simulations of the velocity field in the $(x, y)$-plane at $z=0$ with the multi-holed baffle plate. Some streamlines are shown in the simulation data.

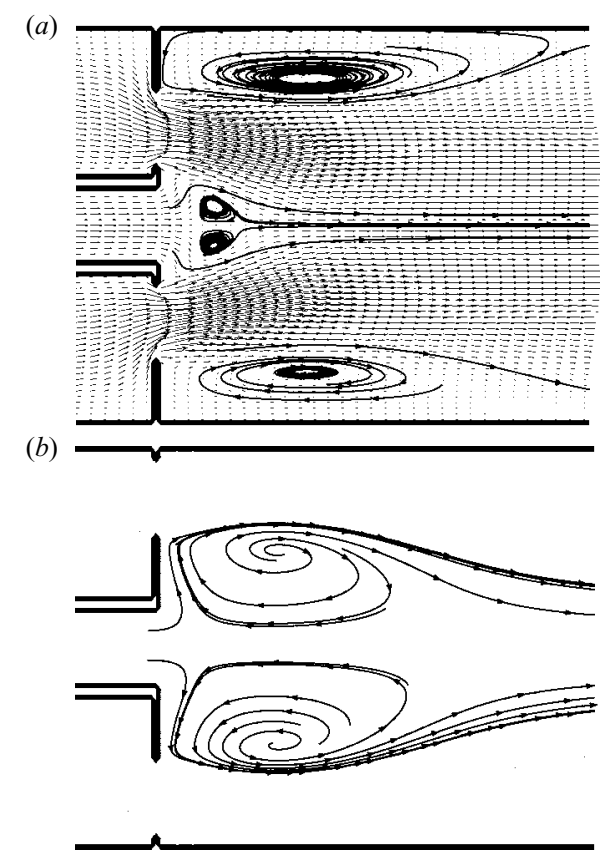

FIGURE 8 . Velocity field in the $(x, y)$-plane at $z=0$ with the multi-holed baffle plate. $(a)$ Holes of the baffle plate are close to the inner tube; $(b)$ The holes are moved away from the inner tube.

reproduce the flow in the microreactor. Figure 8 shows two computed velocity fields for two different configurations of the baffle plate: one with the holes moved radially toward the inner tube and the other one with holes closer to the outer tube wall 

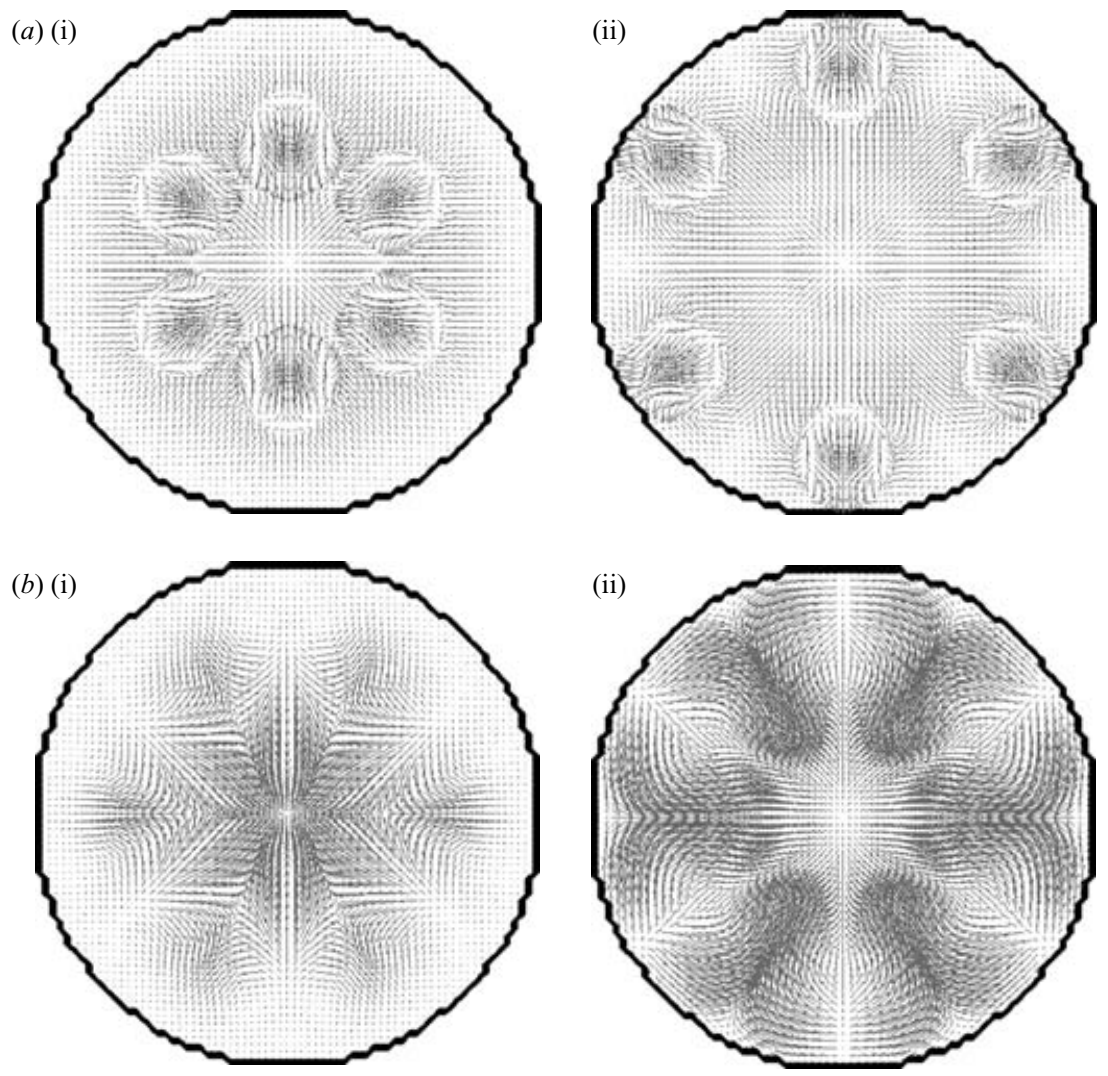

FIGURE 9. Velocity field at $(a) x / d_{1}=0.5,(b) x / d_{1}=5$. (i) Data are from figure $7(a)$; (ii) data are from figure $7(b)$.

$\left(R_{1} / D=0.26\right.$ and 0.4 , respectively; $R_{1}$ is the separation between the inner tube axis and the radial location of the centre of the holes, see figure 1). The figure shows that stationary recirculatory motions take place behind the baffle plate. These results are in excellent agreement with the numerical results of Woodfield et al. (2003) and the experimental data of Moghtaderi et al. (2006). The velocity fields in figure 8 illustrate the effect the location of the baffle plate holes has on the vortical structures. For example, notice the relatively small recirculatory motions near the central tube in case (a) as opposed to the large ones in case $(b)$. There are also elongated and relatively large vortical structures in case $(a)$ near the wall of the main vessel, but they hardly contribute to the mixing between the oxidizer and fuel streams. In case $(b)$, the large recirculatory motions help redirect the fluid issuing from the inner tube towards the outer fluid, which should increase the contact area between the two fluid streams.

Figure 9 shows the same velocity fields as in figure 8 in the $(y, z)$-plane at two streamwise positions $\left(x / d_{1}=0.5\right.$ and 5$)$. Note that for visibility, the velocity vectors have been scaled up by a factor of 100 relative to the data of figure 8 . The baffle plate induces secondary motions in the radial direction, which should further contribute to enhance mixing. Moving the holes outward increases the strength of these secondary motions.

The above results suggest that mixing in laminar flow can be enhanced by creating coherent stuctures. These structures, which generate radial motions may help maintain 
(a)

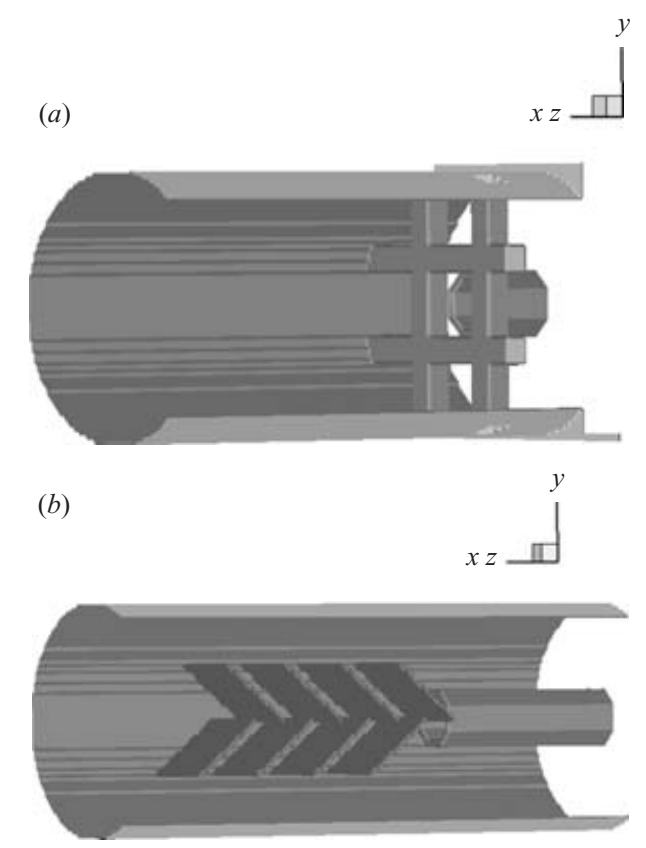

Figure 10. Microreactor with (a) $2 \times 2$ square bars and (b) flat plates.

longer (in space and/or in time) contact between two streams of fluids. However, it is worth observing the strong symmetrical feature of the flow as seen in figure 9. It will be seen later that such symmetry may prove to be disadvantageous to effective mixing.

It is then evident that the multi-holed baffle plate is not the only passive device that can generate such coherent structures. Two other passive means are investigated and compared with the multi-holed baffle plate in the next section. It should be emphasized that only simple geometries for the passive devices are investigated. Considering the smallness of the microreactors, complex geometries, while potentially more efficient than their simple counterparts, are likely not to be used because of the difficulties associated with their fabrication.

\subsubsection{Comparative study between three passive devices}

Two types of passive device for mixing enhancement were studied numerically: a set of $2 \times 2$ square bars and flat plates mounted as shown in figure 10. The former arrangement was selected because bars can generate vortical structures in their wake and having crossed bars may increase interaction between the structures. The flat-plate arrangement was used to divert the fluid from the inner tube towards the outer fluid in order to increase contact between the two fluids. Also the introduction of the flat plates breaks up the geometrical symmetry of the microreactor. This fact is important and is discussed later. The results with the multi-holed baffle plate were used as reference data and we include them in the discussion. Since the inlet conditions were keept constant for the three cases studied $\left(U_{\text {in }}=0.05\right.$ and $V_{\text {in }}=W_{\text {in }}=0 ; U_{\text {out }}=0.02$ and $\left.V_{\text {out }}=W_{\text {out }}=0\right)$, the mass flow rate ratio, $\dot{m}_{\text {in }} / \dot{m}_{\text {out }}\left(\dot{m}_{\text {in }}\right.$ and $\dot{m}_{\text {out }}$ are the mass flow rates for the inner and outer tubes, respectively), changed with the geometry of the passive device (see table 1). The multi-holed baffle plate shows the highest ratio 


$\begin{array}{lclr} & \dot{m}_{\text {in }} / \dot{m}_{\text {out }} & V_{\text {avg }} & R_{D} \\ \text { Baffle plate } & 4.6 & 0.0235 & 94 \\ 2 \times 2 \text { bars } & 3.6 & 0.027 & 109 \\ \text { Plate } & 3.2 & 0.0286 & 116\end{array}$

TABLE 1. Mass flow rate ratios, average velocities and Reynolds numbers. The latter two are calculated for the outer diameter downstream of the inner tube outlet, at $x / d_{1}=30$.

because of the reduced amount of oxidizer flowing through the holes; this is the result of the blockage effect induced by the baffle plate.

Whereas the $2 \times 2$ square bar arrangement maintains some degree of geometrical symmetry, the flat plate arrangement disrupts this symmetry. It should be stressed that since the study is of an exploratory nature, the present arrangements of the bars and plates (spacing, inclination size, etc.) are selected as initial arrangements only to determine their ability to enhance mixing as an alternative to the multi-holed baffle plate. A systematic parametrical study, which is beyond the scope of the present work, would need to be carried out to find the optimum arrangement for each configuration.

Since the inlet conditions are identical for the three configurations, table 1 indicates that the plate arrangement presents the lowest blockage effect. The reduction in the mass flow rate was due mainly to the increase of the outer flow rate. In terms of energy consumption, this shows that the plate arrangement would require less energy than the baffle plate configuration for a given mass flow rate.

Figure 11 shows the velocity field in a vertical plane $(x, y)$ at $z=0$ for the three configurations. Both the multi-holed baffle plate and the $2 \times 2$ bar arrangements generate circulatory motions. The vortices behind the baffle plate appear to be stronger and of larger dimensions than those behind the bars. No such recirculatory structures are observed with the flat-plate arrangement. At first, one might conclude that the latter configuration is less effective than the other two, owing to a lack of vortical structures. However, the deviation of the inner fluid towards the outer fluid caused by the first two flat plates, contributes significantly to mixing enhancement.

While vortical structures are desirable in the laminar regime to increase mixing between two fluid streams, their geometries, orientations with respect to the main flow direction and interactions (e.g. merging, self-entrainment) are crucial for effective mixing. This is illustrated well by the three present cases. Figure 12, shows views of the cross-section of the reactor where two series of streaklines are represented. Both series start at $x / d_{1}=-0.4$ (upstream of the flow manipulator); the first series of streaklines has its 'emission' points aligned vertically, while the 'emission' points for the second series of streaklines are aligned along a line rotated by $45^{\circ}$ with respect to the vertical direction. In the case of the $2 \times 2$ square bar arrangement, the streaklines show no azimuthal deviation. While the multi-holed baffle plate does induce some azimuthal movements, the flat plates produce the most dramatic effect in that respect. This suggests that fluid particles in the multi-holed baffle plate and the $2 \times 2$ square bar arrangements, trapped in the recirculation regions, have little chance of escaping and travelling to other locations. Figure 13 shows some pathlines for inner and outer fluid particles. The reactor wall and the passive mixers are represented (grey) for better visualization. After travelling in the vortical structures behind the multi-holed baffle plate, the particles of the inner fluid return and remain in the region around the centreline; the $(z, y)$ view shows that this region is mainly a cylindrical tube of diameter $d_{1}$ (the diameter of the the inner tude). The outer fluid remains mostly 


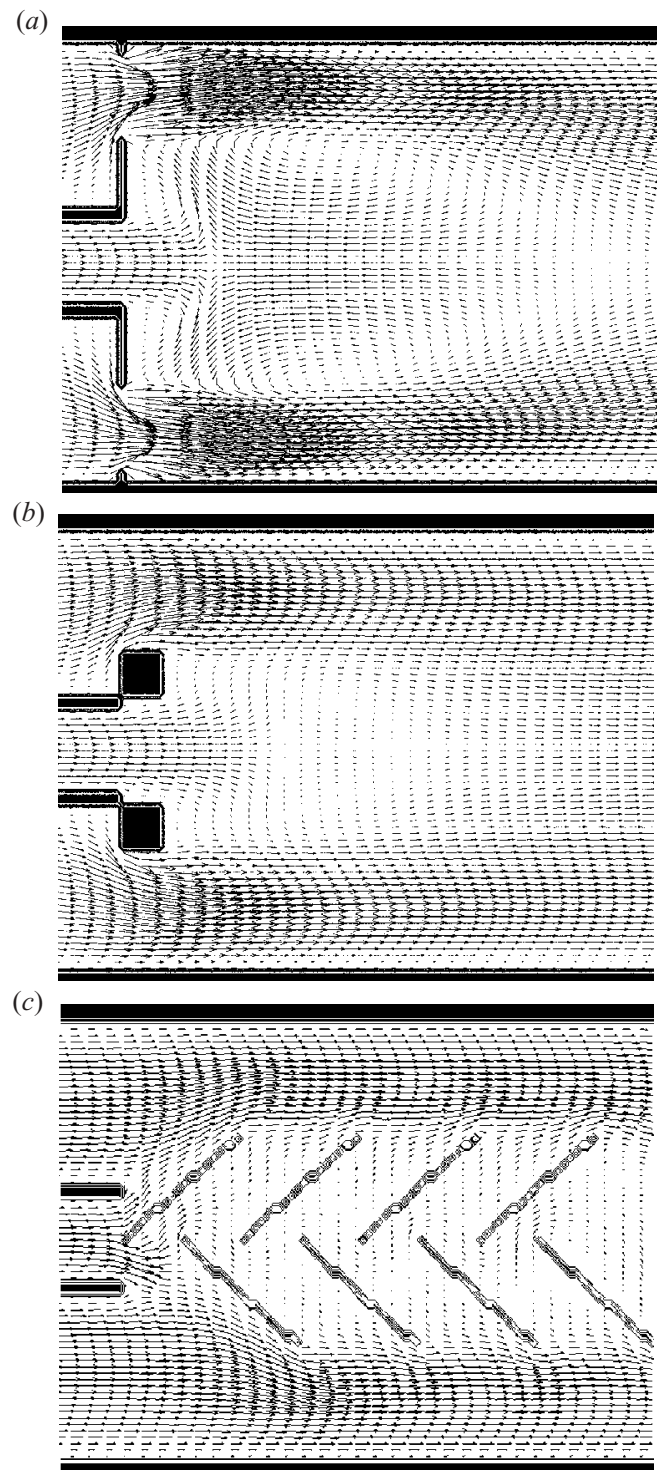

FIGURE 11 . Velocity field in the $(x, y)$-plane at $z=0$ for the three passive devices. (a) Multi-holed baffle plate, (b) $2 \times 2$ square bars; (c) flat plates.

outside of the inner fluid, i.e. outside the cylindrical tube. Figure 13 also reveals that the outer fluid does not flow into the vortical structures. Thus, the outer fluid appears to remain always on the periphery of the inner fluid throughout the microreactor. There is no mingling, or very little, of the two streams, nor is there any folding of the interface between the two fluids. In the case of the flate-plate arrangement, the inner and outer fluid particles show a better blending, as illustrated in the view in the $(z, y)$-plane of figure 13, highlighting an improved mixing relative to the multi-holed baffle plates. Layers of outer fluid are now surrounded by inner fluid downstream of the flate plates. This striation, which results from a stretching and folding process (a combination of strain and rotation), is a clear signature of an efficient mixing. None 

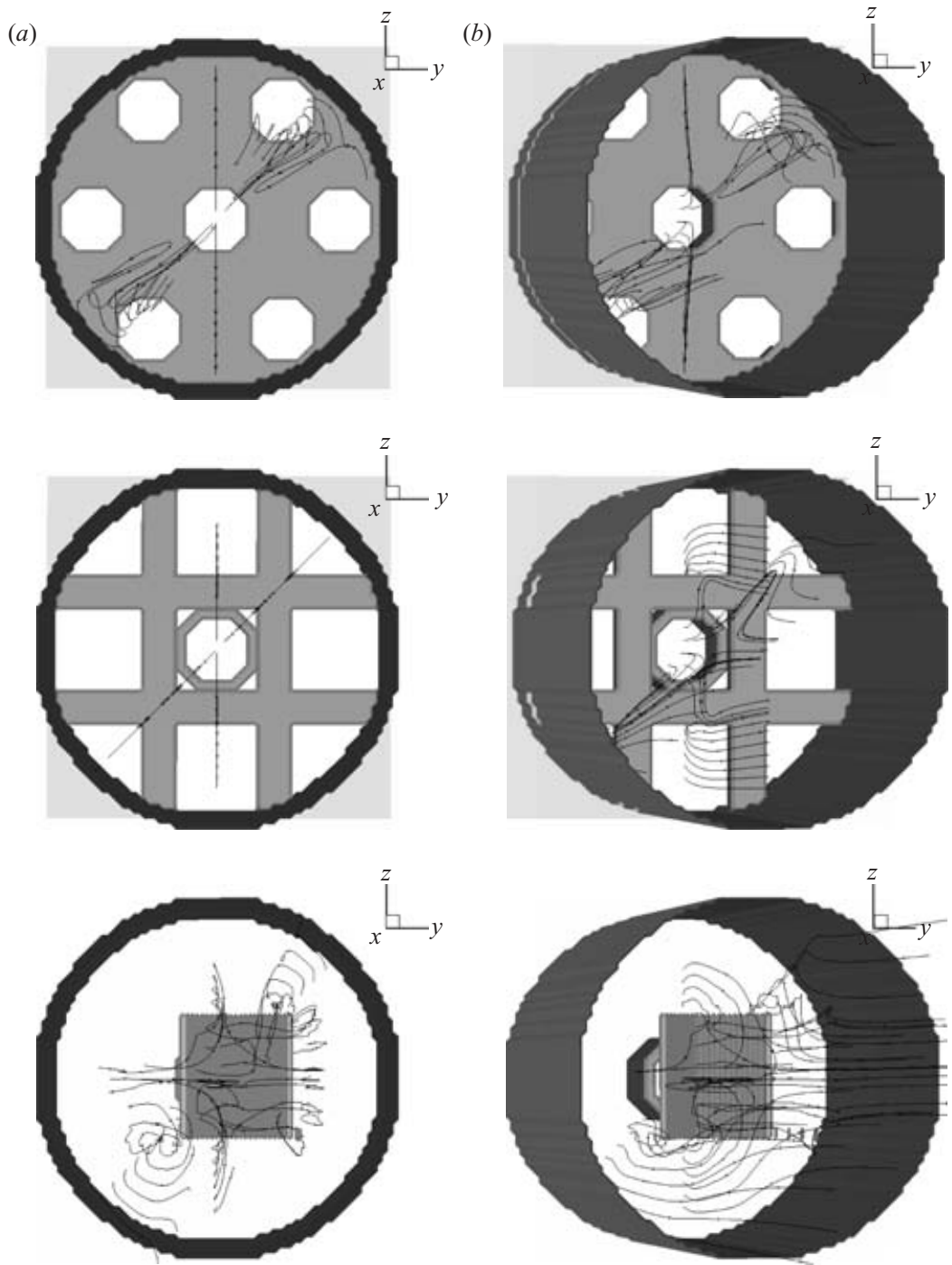

FIGURE 12 . Views of the streaklines in the $(y, z)$-plane. The views in $(b)$ are the same as those in $(a)$, but slightly tilted to the left.

of the other cases presents such striation. Thus, one expects the flat-plate arrangement to promote molecular diffusion more effectively than the other two arrangements.

An important observation that can be made from the above results is that while vortical structures are required ingredients for increasing molecular diffusion, through an increase in interfaces between streams, not all of their features are necessary; somes may be disadvantageous. Characteristics of the vortical structures that may hinder the performance of a mixer are their coherence (or the degree of coherence), their limited extent (or size) and stationarity (or lack of non-stationarity). As it can be seen in the case of the multi-holed baffle plate and the $2 \times 2$ bars, the structures are both strongly coherent, localized in one region of the flow domain and stationary. This tends to trap the fluid particles within the vorticle structures, thus, preventing them from being transported throughout the domain of the flow 


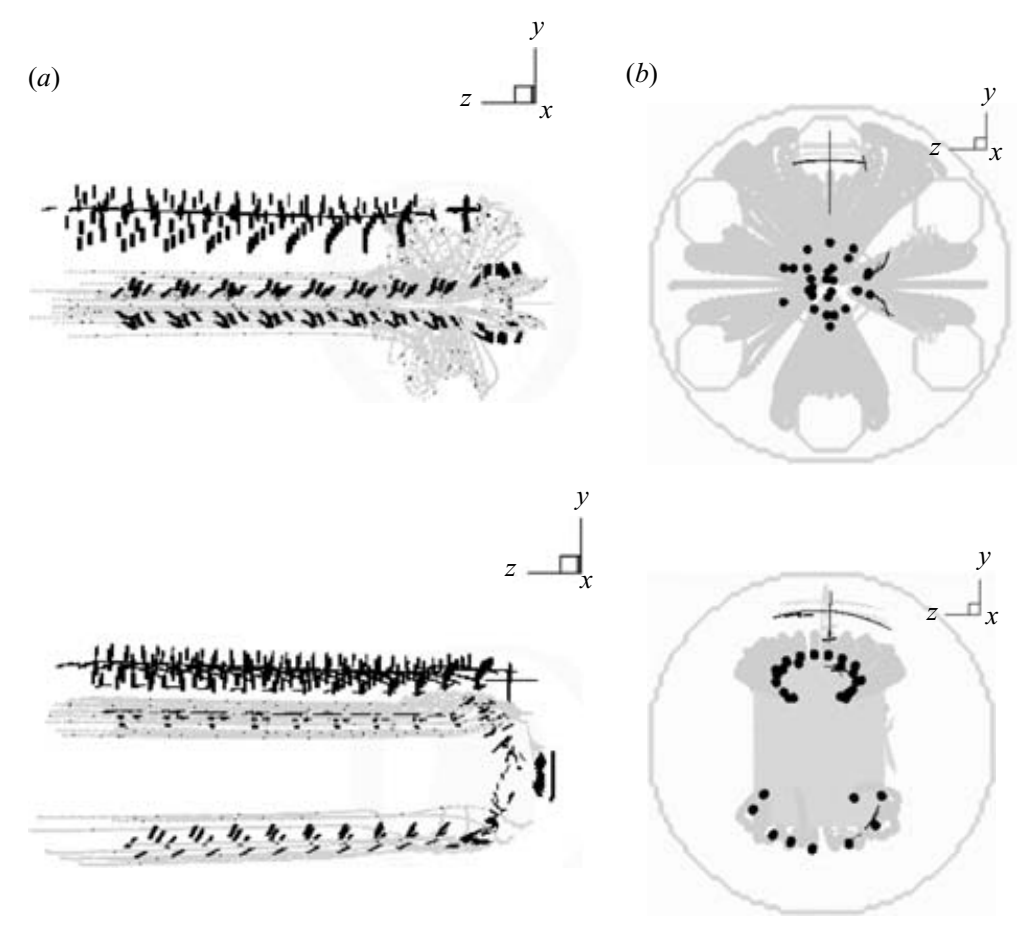

Figure 13. Particles pathlines. ( $a$ ) Perspective view; $(b)$ view in the $(z, y)$-plane at $x / d_{1}=45$. ๑, inner fluid particles; -, outer fluid particles.

and, consequently, resulting in a poor mixing efficiency. We would like to have the fluid particles visit, ideally, the whole domain of the flow so molecular diffusion will be best promoted. The flat-plate arrangement seems to offer a better prospect for fluid particles to visit more regions of the reactor than the multi-holed baffle plate and the $2 \times 2$ square bars. To further support this, figure 14 shows examples of pathlines projected onto the $(y, z)$-plane for the multi-holed baffle plate and the flat-plate arrangements. Two pathlines are represented in each case: one pathline for the inner fluid, and one for the outer fluid. The originating points for the pathlines are kept the same for both arrangements. Notice how the pathlines for the baffle plate present little azimuthal deviation indicated by the inclination of the lines, almost about $45^{\circ}$. In the flat-plate case, the pathlines show a better 'exploration' of the space. Furthermore, the pathlines, which are initially well separated come within close range of each other, in contrast to the case of the baffle plate. Again, this indicates that the flate-pate arrangement provides better contact between the inner and outer fluids, thus promoting better molecular diffusion than the multi-holed baffle plate.

On the basis of figures 12 and 14, one may argue that the flat-plate configuration introduces some degree of 'laminar chaos' or 'chaotic advection' (Ottino 1990; Xia et al. 2005) in the fluid particle paths, thus increasing mixing relative to the other two configurations. However, since the present flow is steady, which means that the velocity field is independent of time (the pathlines, streamlines and streaklines are equal and time independent), care must be taken to use chaotic tools for quatifying the mixing efficiency in the present flow. A steady three-dimensional incompressible flow can be mapped onto a one-degree-of-freedom time-dependent Hamiltonian system 


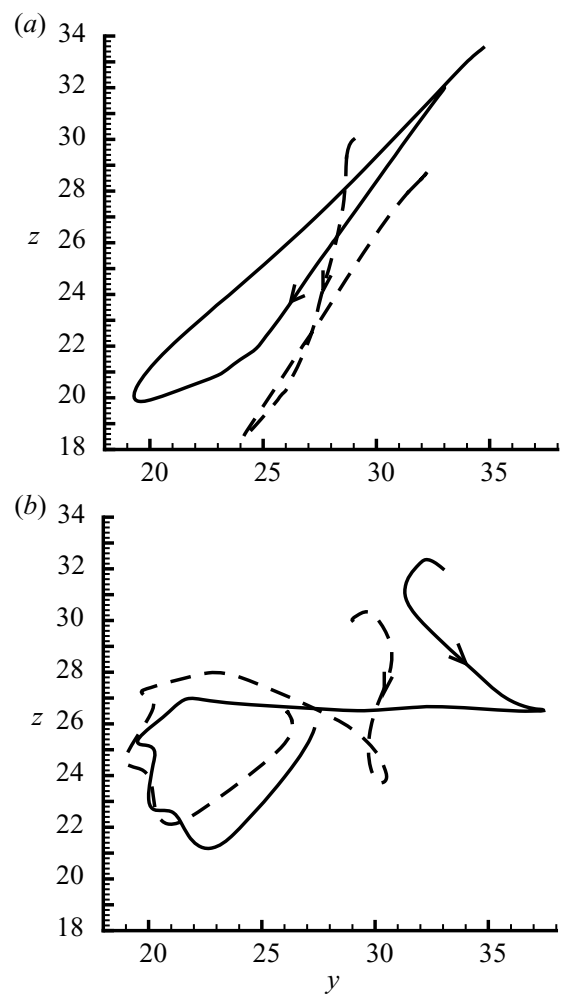

FIGURE 14. Projection of pathlines onto the $(y, z)$-plane. $(a)$ Multi-holed baffle plate; $(b)$ flat plates. ---, outer fluid pathline; _- inner fluid pathline. The arrow indicates the increasing distance along the $x$-direction.

(Aref et al. 1989). This can be achieved by using the $x$-coordinate as a fictitious time, converting the steady three-dimensional flow into an unsteady flow in two dimensions. However, this requires that the flow velocity along the $x$-direction never vanishes, and no stagnation point occurs in the flow. This is not the case for the multi-holed baffle plate and the $2 \times 2$ square bar arragements, as can be seen in figure 11 , where stagnation points are visible. Clearly, we cannot talk of chaotic advection for these two arrangements. In the flat-plate arrangement, since no stagnation points are seen, one may consider using the $x$-coordinate as fictitious time. Thus, using $x$ as a time coordinate, the Lyapunov exponents for the pathlines were calculated for this arrangement. It was found that all the exponents were negative, revealing that the flow is not chaotic, despite the appearance of the pathlines as seen in figure 12 and 14. However, one may consider carrying out a parametrical study where, for example, the spacing between the plates, the tilt angle and/or the orientation, the number of plates and their dimensions could be systematically varied to determine whether the arrangement of the plates, through a repeated sequence of the swirl in counter-rotating motion as observed in figure 12, can lead to chaotic advection.

In the abscence of chaotic motion, one may try to quantify mixing by calculating the enstrophy defined as:

$$
E=\int_{\Omega}|\nabla \times \boldsymbol{v}|^{2} \mathrm{~d} \Omega
$$



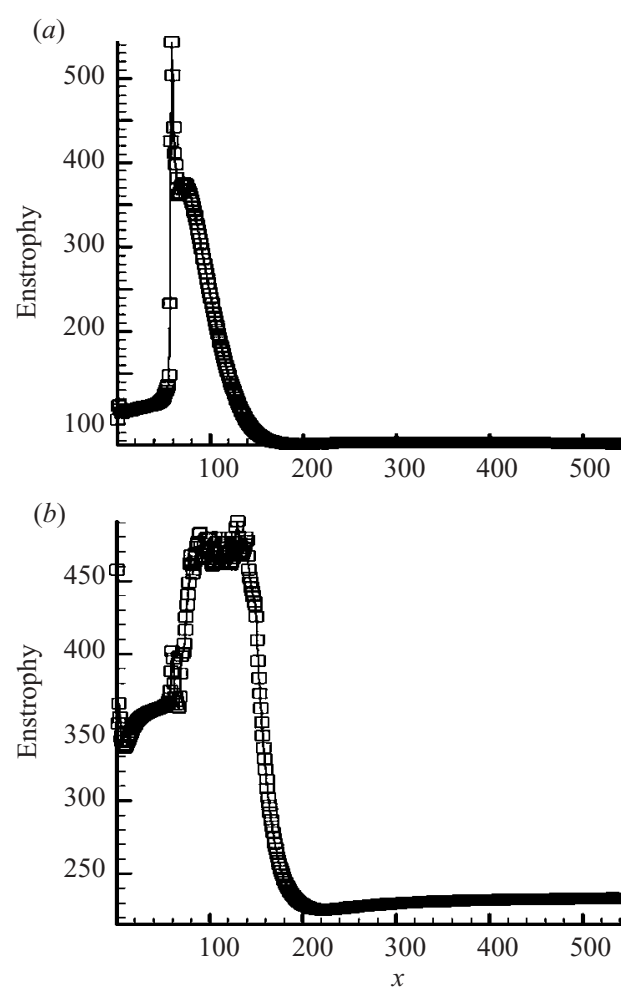

FIGURE 15. Enstrophy variation along the $x$-direction. (a) Muti-holed baffle plate;

(b) flat plates.

where $\Omega$ denotes the region over integration, $v$ is the velocity vector. Figure 15 shows the variation along the $x$-direction of $E$ (where $\Omega$ is the entire plane $(y, z)$ ). It is clear that the flat-plate configuration produces more enstrophy than the multi-holed baffle plate arrangement. Note the strong localized peak for the latter case reflecting the strong velocity gradient at the holes of the baffle plate. The overall increase of enstrophy observed for the flat-plate configuration is due to the vorticity generated at the tip of the plates. This is revealed in figures 16 and 17 showing streamwise vorticity isocontours. Figure 16 shows the isocontour maps at two cross-sections of the microreactor and figure 17 shows two isocontours $\left(w_{x} / w_{x, \max }=0.38\right.$ and -0.38$)$ along the flat plates.

In the multi-holed baffle plate and $2 \times 2$ square bar arrangements, the vortical structures have their axis of rotation perpendicular to the main flow direction, whereas in the case of the flat plates, the vorticle structures tend to have their axis of rotation aligned with the main flow direction. The alignment of the axis of rotation with the main flow direction, which indicates a strong helicity, yields swirling motion. This is seen in figure 18 where the cross-velocity field is shown in a cross-section of the reactor downstream of the flow manipulators. Note the strong radial direction of the velocity, but not much azimuthal motion, in the first two cases. The flow in each case shows a high degree of axial symmetry. In the case of the flat-plate arrangement, distinct vortical motions in the form of longitudinal vortices are visible; there is, however, a plane symmetry in the flow field. The vortical motions are induced by the vortices created at the tips of the plates. This is reminiscent of the tip vortices observed 

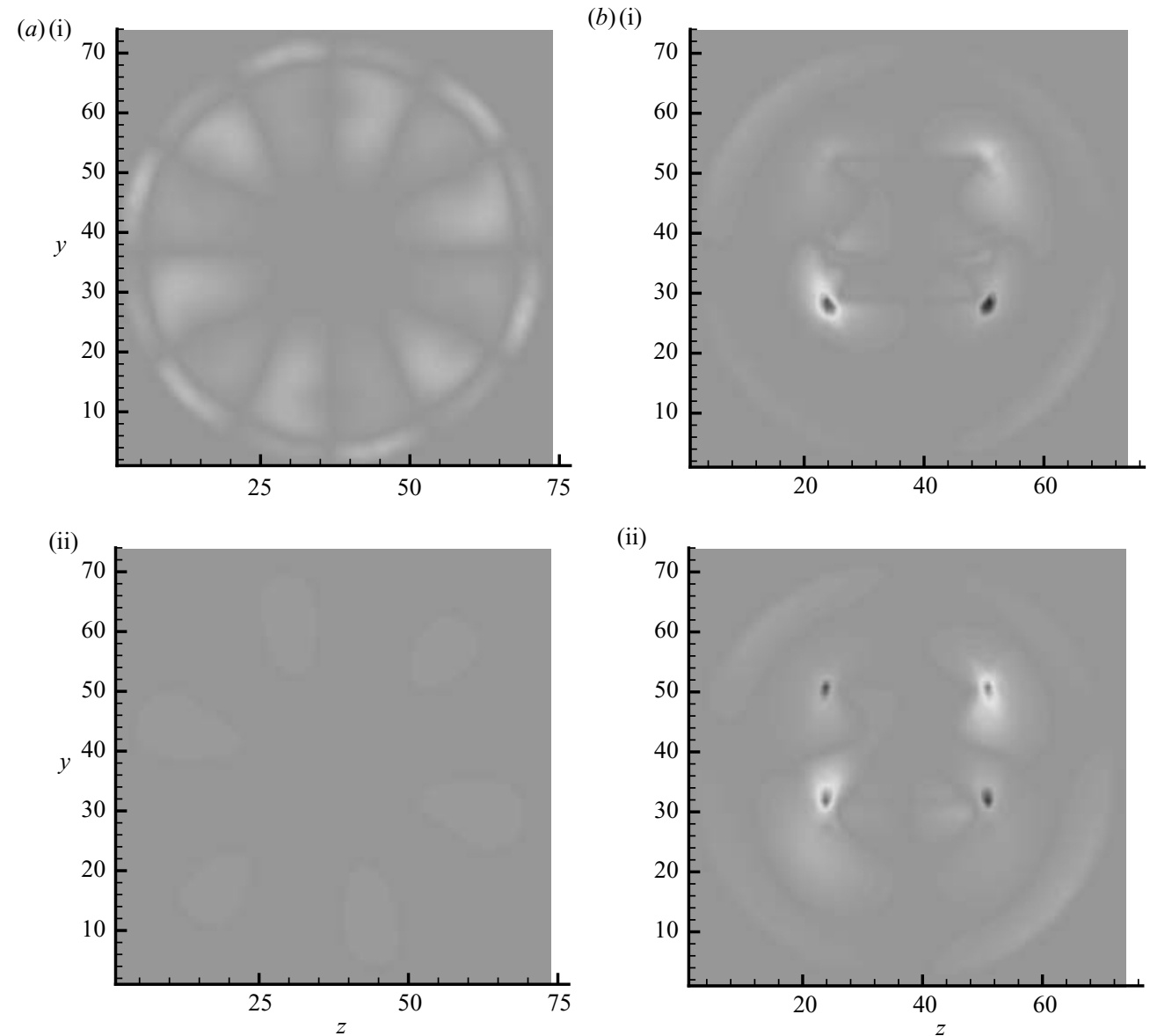

(ii)

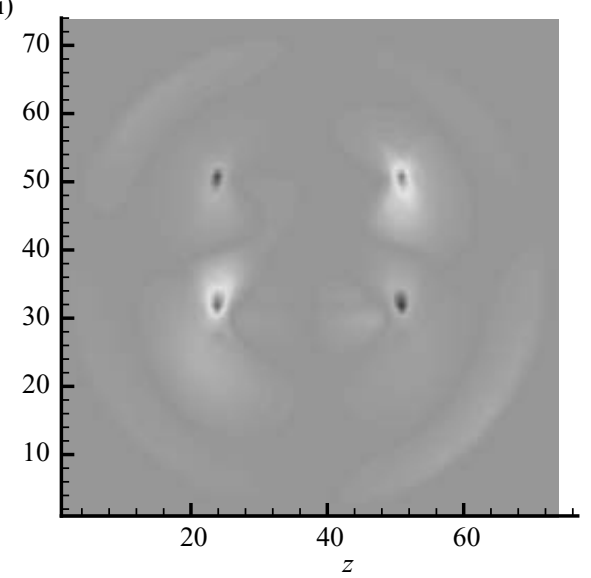

FIGURE 16. Streamwise vorticity. (a) Multi-holed baffle plate; (b) flat plates; (i) $x / d_{1}=1.4$ and (ii) 5.5 .

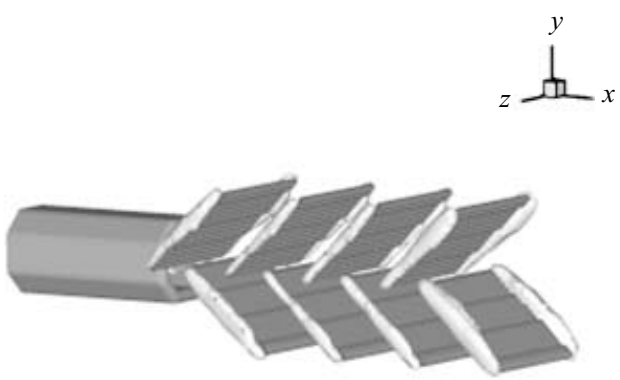

FIGURE 17. Sideview of the inner tube and the flat plates with streamwise vorticity contours (in light colour), $w_{x} / w_{x, \max }=0.38$ and -0.38 , at the tip of the plates.

over a finite wing. However, although tip vortices are generally to be minimized in aviation, here one would rather seek to reinforce them in order to increase the vortical motion, which in turn would improve mixing. In the present case, the tip vortices are created because the plates are at an angle of incidence with respect to the main flow. A systematic study of the effect this angle can have on mixing should be relevant. Also, 

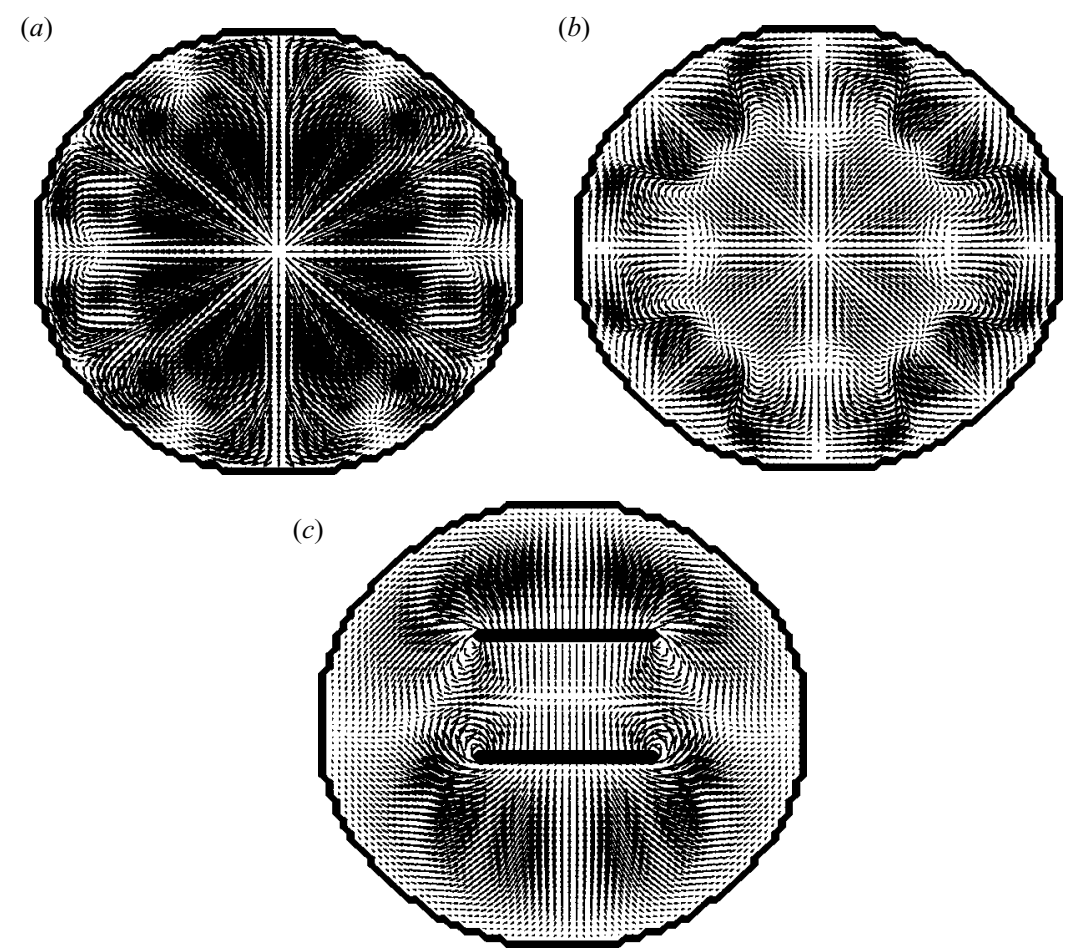

Figure 18. Cross-velocity field in a $(y, z)$-plane at $x / d_{1}=1$ for the three cases. (a) Multi-holed baffle plate; (b) $2 \times 2$ square bars; (c) flat plates.

one may consider using airfoil cross-section shapes for the plates to increase the tip vortices, and determine the best airfoil geometry to optimize mixing. A further study could be carried out on the combination of the flat plates with either the multi-holed baffle or the $2 \times 2$ square bars to further increase laminar mixing. However, care should be taken not to reduce too much the pressure gradient in the reactor.

The above results show that laminar-mixing enhancement in a coaxial microreactor using passive devices can be best optimized when such devices generate flows with coherent structures with no, or at least minimal, symmetrical features. Ideally, the passive devices should induce coherent structures which evolve in time and space, i.e. non-stationary structures. A flow with such coherent structures would present a chaotic pattern, both in space and time, an ideal situation for mixing. Unfortunately, with the range of Reynolds numbers encountered in microreactors, it is almost impossible to generate non-stationary structures. The best one can hope to achieve are structures which, while stationary, show an evolution in space (or spatial deformation), which promotes motion with a minimum of spatial symmetry. This in turn allows fluid particles to visit many regions of the flow domain. In that regard, the tip vortices generated by the flat plates in this study generate motions that improve the wandering of fluid particles of both fluids across the flow domain when compared to the cases of the multi-holed baffle plate and the $2 \times 2$ square bars. It should be emphasized that there may be other passive device geometries that may promote even more mixing than the flat-plate configuration. However, while it is beyond the scope of this paper to investigate more geometries, the present results indicate that the passive devices should induce flows with minimal symmetrical features if the mixing efficiency of the device is to be improved relative to the present ones. 


\section{Concluding remarks}

A lattice Boltzmann simulation of a laminar flow in a coaxial microreactor was carried out with the aim of exploring strategies for laminar-mixing enhancement in the reactor. Three passive mixers were investigated, namely: (i) a multi-holed baffle plate, (ii) $2 \times 2$ square bars, and (iii) flat plates. The results showed that all three devices increased laminar mixing in the microreactor. The physical mechanism which increased mixing is related to the generation of coherent structures in the flow. The structures not only bring into contact the two fluids issuing from the inner and outer tubes of the microreactor, but they also increase the interfacial area between the fluids.

There are, however, important differences in the coherent structures generated by the three devices, which affect the mixing enhancement. For example, the first two passive devices generated a flow structure with strong axial symmetrical features in the cross-section plane; a plane symmety was found in the flow structure induced by the flat-plate arrangement. Also, while the $2 \times 2$ square bars produced virtually no azimuthal flow motion and the multi-holed baffle plate produced such motion marginally, the flat-plate arrangement yielded the most dramatic effect in that respect, thus adding swirl components to the flow. The swirl is created by the downstream entrainment of the tip vortices generated at the edges of the flat plates, which act like finite wings.

It was also observed that the flat-plate arrangement had the largest mass flow rate as compared with the other devices. This indicates that the loss in pressure gradient is minimized with this arrangement. Accordingly, less energy would be required to operate the microreactor with the flat plates than with the multi-holed baffle plate or the $2 \times 2$ square bars.

\section{REFERENCES}

Agrawal, A., Djenidi, L. \& Antonia, R. A. 2005 Simulation of gas flow in microchannels with a sudden expansion or contraction. J. Fluid Mech. 530, 135-144.

Aref, H., Jones, S. W., Mofina, S. \& ZawadzKi, I. 1989 Vortices, kinematics and chaos. Physica D 37, 423-440.

Benzi, R., Succi, S. \& Vegassola, M. 1992 The lattice Boltzmann equation: Theory and application. Phys. Rep. 222, 145-197.

Chen, S. \& Doolen, G. D. 1998 Lattice Boltzmann method for fluid flows. Annu. Rev. Fluid Mech. 30, 329-364.

Deval, J., Tabeling, P. \& Ho, C. M. 2002 A dielectrophoretic chaotic mixer. IEEE 36-39.

DJENIDI, L. 2006 Lattice Boltzmann simulation of a grid-generated turbulence. J. Fluid Mech. 552, $13-35$.

Ehrfeld, W., Hessel, V. \& Loww, H. 2000 Microreactor: New Technology for Modern Chemistry. Wiley-VCH.

Evensen, H. T., Meldrum, D. R. \& Cunningham, D. L. 1998 Automated fluid mixing in glass capillaries. Rev. Sci. Instrum. 69, 519-526.

Frisch, U., Hasslacher, B. \& Pomeau, Y. 1986 Lattice-gas automata for the Navier-Stokes equations. Phys. Rev. Lett. 56, 1505-1508.

Higuera, F. \& Jimenez, J. 1989 Boltzmann approach to lattice gas simulations. Europhys. Lett. 9, 663-668.

Higuera, F., Succi, S. \& Benzi, R. 1989 Lattice gas dynamics with enhanced collisions. Europhys. Lett. 9, 345-349.

Ho, C. M. \& TAI, Y. C. 1998 Micro-electro-mechanical systems (MEMS) and fluid flows. Annu. Rev. Fluid Mech. 30, 579-612.

Karniadakis, G. E. \& BeskoK, A. 2002 Microflows - Fundamentals and Simulations. Springer.

Lee, Y. K., Deval, J., Tabeling, P. \& Ho, C. M. 2001 Choatic mixing in electrokinetically and pressure driven micro flows. IEEE 483-486. 
Moghtaderi, B., Shames, I. \& Djenidi, L. 2006 Microfluidic characteristics of multi-holed baffle plate micro-reactor Intl J. Heat Fluid Flow (in Press).

Nguyen, N. -T. \& Werely, S. T. 2002 Fundamentals and Applications of Microfluidics. Artech House, Boston.

Ottino, J. M. 1990 Mixing, chaotic advection and turbulence. Annu. Rev. Fluid Mech. 22, 207-253.

Ottino, J. M. \& Wiggins, S. 2004 Phil. Trans. R. Soc. Lond. A 362, 923-935.

Rossi, N., Ubertini, S., Bella, G. \& Succi, S. 2005 Unstructured lattice Boltzmann method in three dimensions. Intl J. Numer. Meth. Fluids 49, 619-633.

Stone, H. A., Strook, A. D. \& Ajdari, A. 2004 Engineering flows in small devices: microfluidics towards a lab-on-a-chip. Annu. Rev. Fluid Mech. 36, 381-411.

SuccI, S. 2001 The lattice Boltzmann equation for fluid dynamics and beyond. Numerical Mathematics and Scientific Computation. Oxford University Press.

Suzuki, H. \& Ho, C. M. 2002 A magnetic force driven chaotic micro-mixer. IEEE 40-43.

Verpoorte, E. \& RooiJ, N. F. D. 2003 Microfluidics meets MEMS. IEEE 91, 930-953.

Woodfield, P. L., KazuYoshi, N. \& Suzuki, K. 2003 Numerical study for enhancement of laminar flow mixing using mutiple confined jets in a micro-can combustor. Intl J. Heat Mass Transfer 46, 2655-2663.

Xia, H. M., Wan, S. Y. M., Shu, C. \& Chew, Y. T. 2005 Chaotic micromixers using two-layer crossing channels to exhibit fast mixing at low Reynolds numbers. Lab Chip 5, 748-755. 